\title{
Post-Aire maturation of thymic medullary epithelial cells involves selective expression of keratinocyte-specific autoantigens
}

\author{
Xiaoping Wang ${ }^{1+\ddagger}$, Martti Laan ${ }^{1 *}{ }^{*}$, Rudolf Bichele ${ }^{1}$, Kai Kisand ${ }^{1}$, Hamish S. Scott ${ }^{2,3,4}$ and Pärt Peterson ${ }^{1}$ \\ ${ }^{1}$ Molecular Pathology, Institute of General and Molecular Pathology, University of Tartu, Tartu, Estonia \\ 2 Department of Molecular Pathology, The Centre for Cancer Biology, SA Pathology, Adelaide, SA, Australia \\ ${ }^{3}$ The School of Medicine, University of Adelaide, Adelaide, SA, Australia \\ ${ }^{4}$ The School of Molecular and Biomedical Science, University of Adelaide, Adelaide, SA, Australia
}

\section{Edited by:}

Adrian Liston, Vlaams Instituut voor

Biotechnologie, Belgium

\section{Reviewed by:}

Mitsuru Matsumoto, University of

Tokushima, Japan

Daniel Gray, The Walter and Eliza Hall Institute for Medical Research,

Australia

James Dooley, Vlaams Instituut voor

Biotechnologie, Belgium

Olga Ucar, German Cancer Research

Center, Germany

${ }^{*}$ Correspondence:

Martti Laan, Molecular Pathology, Institute of General and Molecular Pathology, University of Tartu, Ravila 19, Biomedicum, Tartu 50414, Estonia. e-mail:martti.laan@ut.ee

\section{${ }^{\dagger}$ Present address:}

Xiaoping Wang, Department of

Pathology, Shaanxi University of

Chinese Medicine, Xianyang 712046,

Shaanxi, China.

e-mail:wxpphd@yahoo.com.cn

${ }^{\ddagger}$ Xiaoping Wang and Martti Laan have contributed equally to this work.
The autoimmune regulator (Aire)-directed ectopic expression of tissue-specific antigens (TSAs) by mature medullary thymic epithelial cells (mTECs) has been viewed as an essential mechanism in the induction of central tolerance. Recent data suggest that the survival of mTECs extends beyond the Aire+ cell population to form the post-Aire mTEC population and Hassall's corpuscles (HCs). The nature and function of these post-Aire epithelial cells and structures, however, have remained unidentified. In this study, we characterized in detail the end-stage development of mTECs and HCs in both Aire-sufficient and Airedeficient mice. In addition, using a transgenic mouse model in which the LacZ reporter gene is under the control of the endogenous Aire promoter, we purified and analyzed the postAire mTECs to characterize their function. We showed that the end-stage maturation of mTECs closely resembles that of keratinocytes and that the lack of Aire results in a marked block of mTEC differentiation, which is partially overcome by ligands for RANK and CD40. We also provide evidence that, during mTEC development, Aire is expressed only once and during a limited 1-2 day period. The following loss of Aire expression is accompanied by a quick downregulation of MHC class II and CD80, and of most of the Aire-dependent and Aire-independentTSAs, with the exception of keratinocyte-specific genes. In the final stage of maturation, the mTECs lose their nuclei to become HCs and specifically express desmogleins (DGs) 1 and 3, which, via cross-presentation by APCs, may contribute to tolerance against these pemphigus vulgaris-related TSAs.

Keywords: Aire, thymus, Hassall's corpuscle, thymic medullary epithelial cells, central tolerance, desmoglein, autoantigen

\section{INTRODUCTION}

The thymus is the primary lymphoid organ involved in the development of thymocytes and has a fundamental role in establishing immune tolerance (Gallegos and Bevan, 2006). Impaired thymic clonal deletion or ineffective functional inactivation of self-reactive $\mathrm{T}$ cells can lead to a breakdown of central tolerance and the development of autoimmune diseases.

An essential feature in the induction of central tolerance is the promiscuous expression of tissue-specific antigens (TSAs) by terminally differentiated medullary thymic epithelial cells (mTECs; Derbinski et al., 2001). The pool of these mTEC-expressed TSAs is extremely diverse and covers most of the putative autoantigen targets of experimental animal models and of human autoimmune diseases (Kyewski and Derbinski, 2004). As mTECs also highly express MHC molecules with co-stimulatory signals, the TSAs can be directly presented to the developing thymocytes, resulting in negative selection, and/or regulatory T-cell (Treg) induction.
A crucial role in the regulation of TSAs has been attributed to the autoimmune regulator (Aire; Anderson et al., 2002). Being induced by the tumor necrosis factor receptor superfamily (TNFRSF) ligands RANKL and CD40L (Rossi et al., 2007; Akiyama et al., 2008), Aire is predominantly expressed in the mature (MHCII high, CD80 high) postmitotic mTECs (Derbinski et al., 2005). The lack of Aire in a mouse model is accompanied by the downregulation of more than a thousand TSAs in the mTECs resulting in multiple defects in thymocyte development including impaired negative selection (Liston et al., 2003), Treg production (Kekalainen et al., 2007; Pomie et al., 2011), and CD4SP cell maturation (Li et al., 2007). The main outcome is autoimmunity against multiple tissues, described both in Aire-deficient mice as well as in AIRE-deficient (autoimmune polyendocrinopathycandidiasis-ectodermal dystrophy) patients (Anderson et al., 2002; Peterson and Peltonen, 2005).

This ability of Aire to regulate the expression of such a large number of genes has drawn interest in understanding the mechanisms behind this control. The prevailing view suggests that Aire 
controls the transcription of TSA expression by preferential binding with the unmethylated form of histone 3 lysine 4 ( $\mathrm{H} 3 \mathrm{~K} 4 \mathrm{me} 0)$, i.e., the inactive mark of transcriptional activity, thus favoring the TSAs, which, in the mTECs, often bear this mark in their promoter region (Peterson et al., 2008; Abramson et al., 2010; Anderson and Su, 2011). However, recent data have pointed out that Aire can also control the maturation of mTECs (Gillard et al., 2007; Dooley et al., 2008; Yano et al., 2008; Milicevic et al., 2010; Matsumoto, 2011), thus suggesting an additional mechanism behind the control of TSA expression.

The maturation of mTECs is phenotypically characterized by the upregulation of MHC class II and CD80 followed by the upregulation of Aire as well as Aire-dependent and Aire-independent TSAs (Gray et al., 2007; Rossi et al., 2007). These class II high, CD80 high, Aire+ mTECs have been in general considered to be postmitotic end-stage mTECs which, after subsequent upregulation of apoptotic signals, will be phagocytosed and removed. On the other hand, there is accumulating evidence that the differentiation of mTECs may continue beyond the Aire+ stages. The first indication came from the presence and nature of Hassall's corpuscles (HCs) in the thymic medulla. These enigmatic tissue structures are believed to contain terminally differentiated mTECs and are characterized by positive staining for markers specific for terminally differentiated upper layers of the epidermis such as cytokeratin (CK) 6, CK10, lympho-epithelial Kazal-type related inhibitor (LEKTI) and involucrin (Nuber et al., 1996; Bodey et al., 2000; Hale and Markert, 2004). Because the Aire KO mice reportedly have no HCs (Yano et al., 2008), and because the development of HCs follows that of Aire+ mTECs during ontogeny (White et al., 2010), it is likely that these epithelial cells represent a postAire cell population. The precise link between Aire+ mTECs and HCs has, however, not been characterized. The function of HCs is also unknown, although their role in Treg induction has been suggested, mostly because of the co-localization of HCs and TSLP in the human thymus (Watanabe et al., 2005).

The second indication of the presence of the post-Aire mTECs came from a recent work that, by using cell fate mapping, directly demonstrated that mTEC differentiation continues beyond the Aire + stage to become an Aire- MHC class II intermediate mTEC population (Nishikawa et al., 2010). Whether these post-Aire mTECs are still capable of promiscuous gene expression/antigen presentation and how this population relates to the HCs, are currently not known.

The aim of this study was to characterize the nature and function of post-Aire mTECs/HCs in mouse thymi and to determine the role of Aire in the late stages of mTEC development. By using a transgenic mouse model in which the Lac $Z$ reporter gene is under the control of the endogenous Aire promoter, we were able to visualize/purify the post-Aire mTEC populations, in order to characterize a direct link between Aire+ cells and HCs and to indicate a unique function for these post-Aire mTEC populations.

\section{RESULTS \\ CHARACTERIZATION OF END-STAGE mTECs/HCs BY EPIDERMAL MARKERS IN MICE}

In order to characterize the final stages of mTEC maturation/HC development in comparison with the maturation of keratinocytes, we evaluated the expression of four (CK6, CK10, involucrin, LEKTI) epidermal cell markers in mouse skin vs. thymus (Figure 1). In the skin, CK6 showed the earliest expression pattern starting from the stratum basale through the stratum spinosum to the stratum granulosum. CK10 was another broadly expressed protein showing strong expression from the stratum spinosum through the stratum granulosum to the stratum corneum. Involucrin showed a much more restricted expression pattern, mostly in the stratum granulosum but also weak staining in the stratum corneum, whereas LEKTI was specifically expressed only in the stratum granulosum. In the thymus, anti-CK6 reacted with a significant number of isolated mTECs but also showed an intense staining in the outer layers of HCs. CK10 showed a fairly similar expression pattern and, in addition, also stained the inner layers of HCs. The expression of involucrin and LEKTI never involved isolated cells and was limited to the outer layers of HCs. We also found very similar expression patterns for all four epidermal differentiation markers in a rat thymus, in which the HCs have been characterized in more detail, thus confirming the true nature of the HCs in mice (Figure A1 in Appendix). In addition, the only antibody that cross-reacted with the human homolog, anti-CK10, selectively stained human HCs (Figure A1 in Appendix). Thus, we confirm that the epidermal differentiation markers can indeed be used for the identification of the end-stage mTEC population as well as for the identification of HCs in mice.

\section{AIRE DEFICIENCY-INDUCED BLOCK IN END-STAGE mTEC MATURATION IS REVERSED BY TNFRSF LIGANDS}

A role of Aire in mTEC maturation has been suggested by showing the lack of HCs as well as involucrin staining in Aire-deficient mice (Yano et al., 2008). The development of involucrin positive HCs, on the other hand, has been shown to depend on signals from mature single positive thymocytes (White et al., 2010). To determine whether this developmental block in the Aire KO mice extends beyond the very late (i.e., involucrin positive) stages of mTEC differentiation, we determined the levels of Aire and HC-markers in wild-type (WT), heterozygous, and Aire-deficient mice. There was a substantial reduction of all four epidermal markers at the mRNA level as well as of CK6 and involucrin at the protein level in the Aire-deficient mice; whereas the changes for protein levels of CK10 and LEKTI in the Aire-deficient mice did not reach statistical significance (Figures 2A,B). However, despite being greatly reduced, some HCs clearly remained even in the Aire KO mice (Figure 2B).

As the development of HCs in the Aire-sufficient mice has been shown to depend on TNFRSF signaling (White et al., 2010), we tested if this requirement also stands for the Aire-independent HCs. Thus, we used ex vivo stimulation of thymic explants from Aire KO mice by LIGHT, RANKL, and CD40L and measured the expression of involucrin thereafter (Figure 2C). Both the protein and mRNA levels of involucrin showed a clear increase after treatment with RANKL or CD40L, suggesting no major differences between the induction of HCs derived from the Aire+ vs. AiremTECs.

THE MAJORITY OF HCs ARE DIRECTLY DERIVED FROM Aire+ mTECs Although being clearly dependent on Aire, we found only 20\% of CK6- or CK10-positive isolated mTECs that co-stained with 


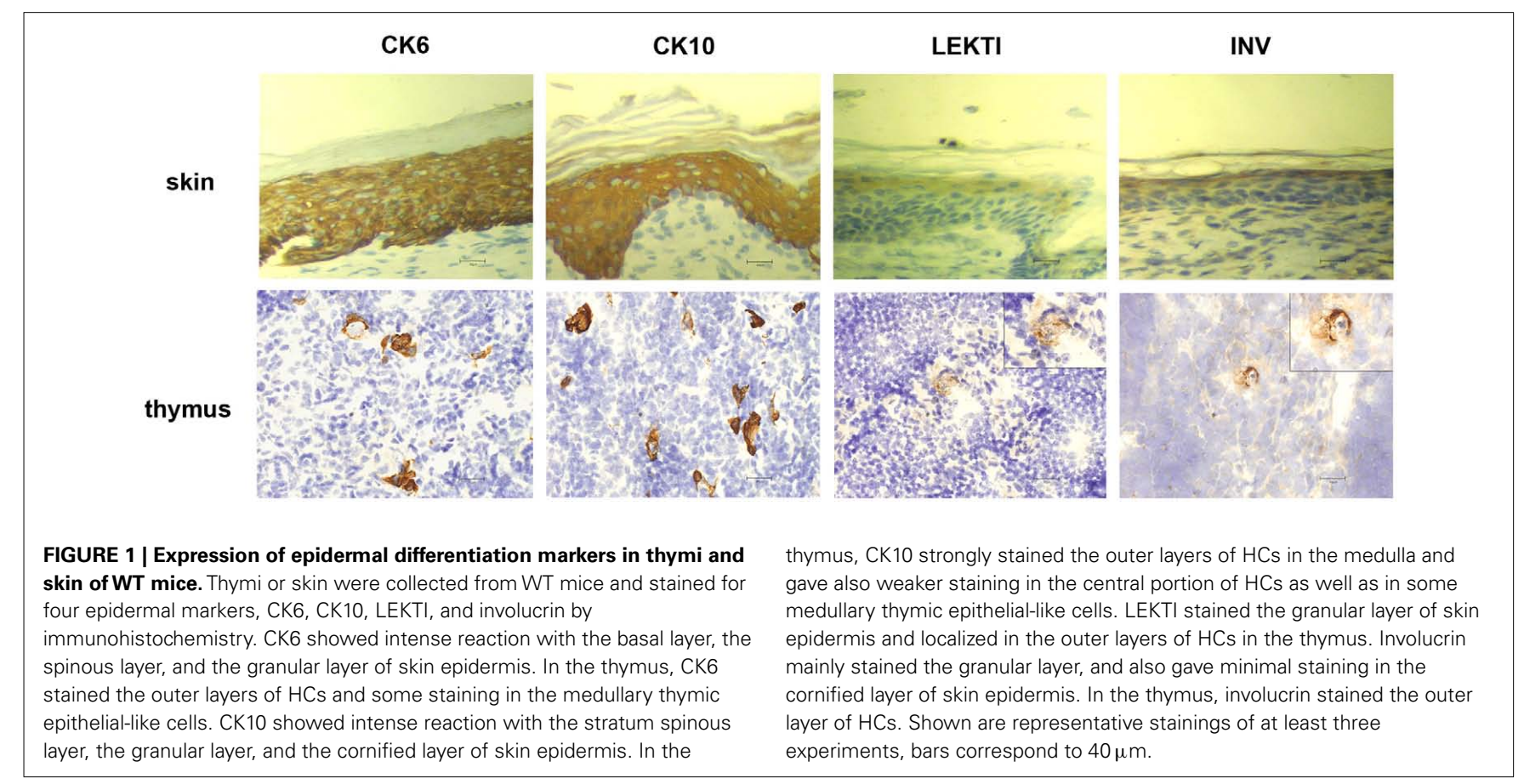

Aire antibody, and virtually no Aire staining was observed in the HCs positive for any of the maturation markers (Figure 2A). On the other hand, Aire has been shown to have a relatively short half-life and can thus be degraded by the time when the mTECs reach the HC-stage of development. To overcome this problem we traced the expression of a more stable protein, LacZ, in a mouse model in which the LacZ reporter gene is under the control of the endogenous Aire promoter, thus creating an Aire-LacZ fusion (Hubert et al., 2009). In this model, in the heterozygous mouse, the expression of Aire can be monitored by the robust enzymatic reaction for LacZ and also by a much more sensitive antibody staining against $\beta$-galactosidase ( $\beta$-gal; Pereira et al., 2006). In parallel with the standard enzymatic LacZ staining, we also used a fluorescent substrate-based detection kit for the enzymatic activity of $\beta$-gal (please see the Materials and Methods for details). In frozen thymic sections from Aire-LacZ heterozygous mice, incubation with this fluorescent substrate resulted in a speckled staining located in the medullary areas and EpCAM positive (medullary) cells, being hence compatible with a signal specific for LacZ enzymatic activity (Figure A2 in Appendix). We could not detect any signal neither for the $\beta$-gal antibody nor for enzymatix LacZ in the thymi from WT mice, further verifying the specificity of the stainings (Figure A3 in Appendix).

Thus, we first confirmed the previously published data (Hubert et al., 2009) showing that in the heterozygous Aire-LacZ mouse both Aire and $\beta$-gal antibody stainings are located in the mTEC nuclei and, as compared to WT Aire staining, tend to form less numerous and larger speckles. However in these Aire-LacZ+/thymi, the majority (mean with SEM: $65 \pm 3, n=3$ ) of Aire+ cells also co-stained for $\beta$-gal (Figure 3A; Figure A3 in Appendix). At the same time, although the enzymatic LacZ staining was $100 \%$ positive for $\beta$-gal by antibody staining, there was absolutely no co-localization of staining for Aire (by antibody) and
LacZ enzymatic activity (Figures 3A,B; Figure A4 in Appendix). This expression pattern was consistent regardless of the detection method (i.e., immunohistochemistry vs. immunofluorescence), strain $(\mathrm{C} 57 \mathrm{BL} / 6$ vs. BALB/c), or age of mice analyzed (see also below). Thus, collectively, these data suggest that the short-lived Aire expression has already disappeared by the time that the enzymatic activity for LacZ and the respective staining develops. To confirm this proposed order of mTEC development, we studied thymi from the same Aire $+/-$ mice during their development from embryonic day (e) 13.5 to day 5 after birth (Figures 3B,C). Aire staining was visible from the day e14.5: at this time there was no expression of LacZ or of any of the maturation markers. The enzymatic LacZ staining developed at e15.5, again with no co-staining with the Aire protein, suggesting the consecutive order of the steps of development for the cells positive for Aire vs. those positive for LacZ. The first mTEC maturation markers, CK10 and involucrin, appeared at embryonic days e15.5 and e18.5, respectively, whereas the morphologically distinct HCs also appeared at e18.5 and increased gradually in size throughout the experiment (Figure 3C).

Because this model allowed us to follow the development of HCs from Aire+ mTECs directly, we also co-stained the thymi from 6-week-old heterozygous mice for LacZ enzymatic activity and the epithelial maturation markers (Figures 3D,E). The majority of involucrin- and LEKTI-positive HCs were also positive for LacZ, thus showing that the majority of HCs indeed develop from the Aire+ mTEC population. The CK10 and CK6 expression was, as already stated above, broader and much less related to the expression of LacZ at a single cell/HC level.

In order to further confirm the lineage connection between Aire+ mTECs and HCs, we used an alternative reporter system, the Aire-driven islet-specific glucose-6-phosphatase-related protein (IGRP)-green-fluorescent protein (GFP; Adig) mouse, 


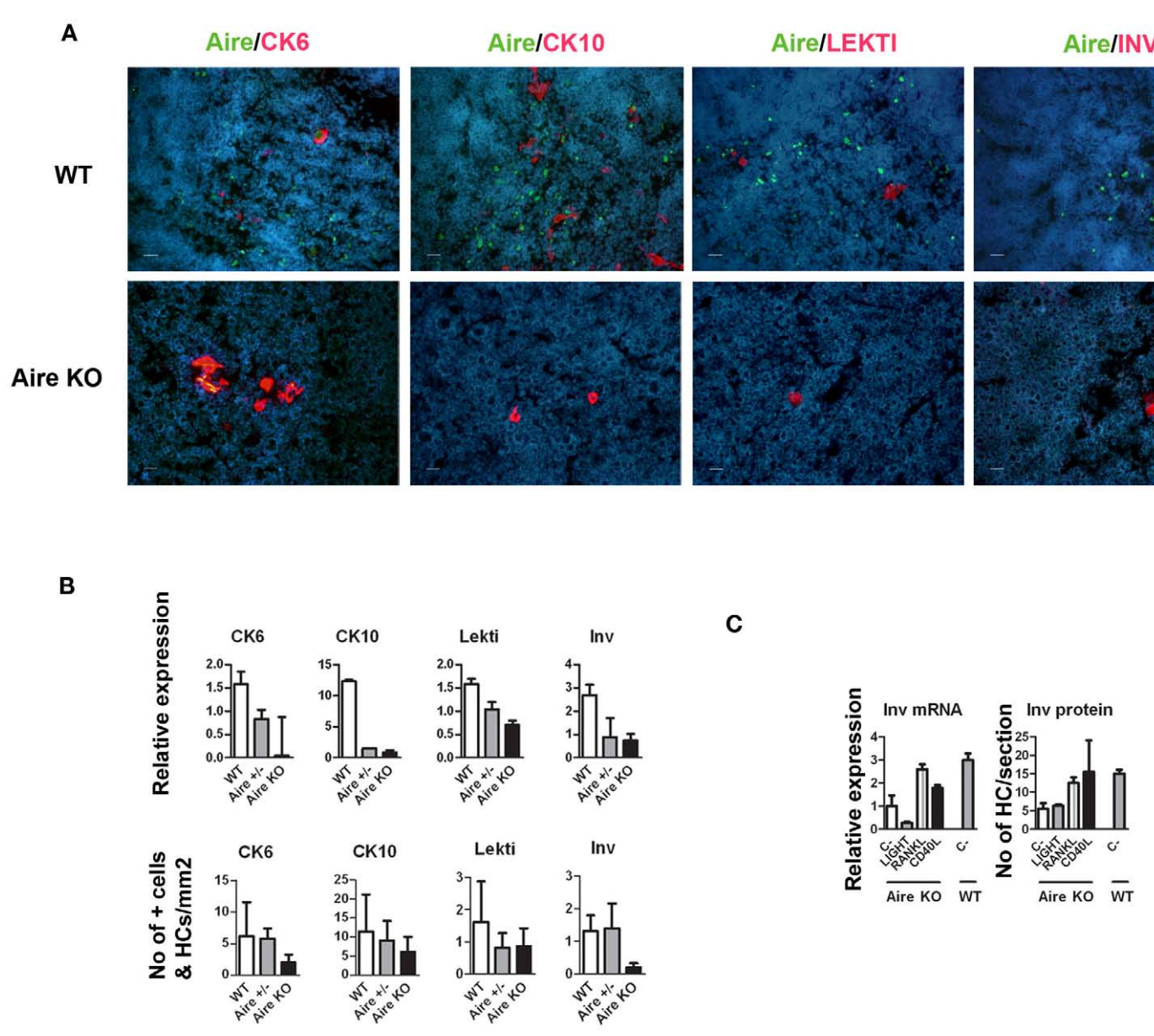

FIGURE 2 | Effect of Aire on the expression of epidermal differentiation markers and the effect of TNFRSF ligands on the expression of involucrin. (A) Thymi from 6-week-old WT or Aire KO mice were stained for Aire and four epidermal differentiation markers CK6, CK10, LEKTI, involucrin by immunofluorescence. In the WT mice, Aire showed green dots in the nuclei of medullary thymic epithelial-like cells. CK6, CK10, LEKTI, and involucrin showed staining mainly in the outer layer of $\mathrm{HCs}$. There was some co-staining for Aire and CK6 or CK10, whereas Aire and LEKTI or involucrin never co-localized. In the Aire KO thymus, there was no staining for Aire, while CK6, CK10, LEKTI, and involucrin showed some staining in the outer layers of $\mathrm{HCs}$. This relatively rare positive staining is depicted on the panels of the Aire KO mice, whereas for the WT mice typical representative medullary stainings of at least three experiments are shown. DAPI was used for nuclear staining. Bars correspond to $20 \mu \mathrm{m}$. (B) Relative gene and protein expression of epidermal differentiation markers in wild-type (WT), heterozygous (Aire ${ }^{+/-}$), and Aire KO mouse. Whole thymi were collected for RNA purification and analyzed by qPCR or stained for CK6, CK10, LEKTI, and involucrin. In the Aire KO mouse, there was a statistically significant reduction of all four epidermal differentiation markers at the mRNA level and of CK6 and involucrin at the protein level ( $p<0.05$ as compared with WT, Student's $t$-test, $n=3$ ), whereas the protein signals for $\mathrm{CK} 10$ and Lekti did not reach statistical significance $(p>0.05)$. Values are mean \pm SEM of triplicate experiments. (C) Relative gene and protein expression of involucrin in Aire $\mathrm{KO}$ thymus after treatment with LIGHT, RANKL, or CD40L. Thymi from Aire KO or WT mice were treated ex vivo for 24 (for mRNA) or $48 \mathrm{~h}$ (protein) and analyzed thereafter for involucrin expression by qPCR or immunofluorescence. The TNFRSF ligands RANKL and CD40L increased the gene and protein expression of involucrin in the Aire KO mice $(p<0.05$, Student's $t$-test, $n=3)$. Values are mean \pm SEM of triplicate experiments. where the Aire+ lineage can be traced by the expression of another very stable protein, GFP (Gardner et al., 2008). We first did the co-stainings for Aire and GFP to confirm the previously published finding that in the heterozygous Adig mouse, the majority (mean with SEM: $84 \pm 6, n=3$ ) of the Aire+ cells stain also positive for GFP (Figure 4A). More importantly, however, we could not find a single involucrin positive HC (Figure 4B; Figure A5 in Appendix), which was not positive for GFP, confirming the direct link between Aire+ mTECs and HCs.

\section{PROMISCUOUS GENE EXPRESSION AND DIRECT ANTIGEN}

PRESENTATION ARE UNIQUE FEATURES OF MHC II Aire+ mTECS THAT ARE LOST OUICKLY DURING FURTHER MATURATION

As the Aire+ mTECs are characterized by their ability to express and present TSAs, we next aimed to evaluate whether these properties are preserved at the post-Aire stage of development. We FACS sorted the post-Aire mTEC population (Figure 5A) based on the presence of LacZ (see Figure A6 in Appendix for additional technical controls) and compared this population's expression profile by quantitative real-time PCR (qPCR) against the LacZ-, MHC class 
A
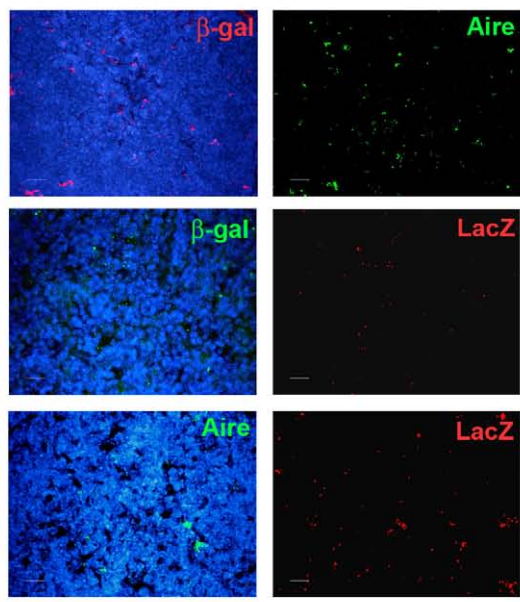

B

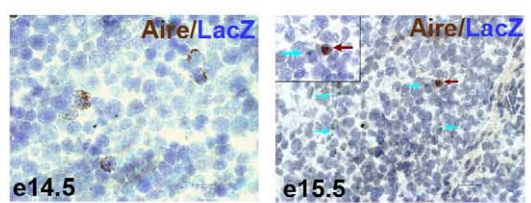

D

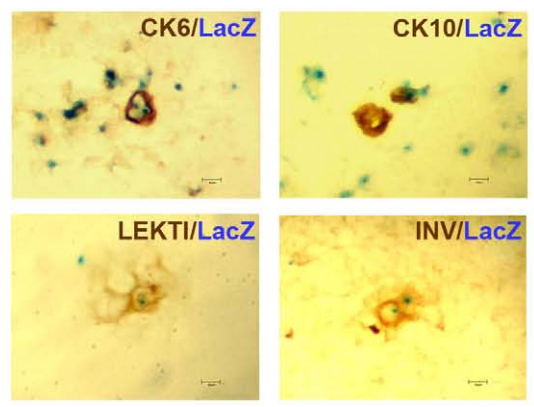

FIGURE 3 | Identification of the post-Aire mTEC population in Aire+/mice. (A) Representative samples of immunofluorescent co-stainings for $\beta$-gal antibody, Aire antibody, and enzymatic LacZ in the thymi from 6-week-old Aire+/- mice. There was no co-localization of Aire and enzymatic LacZ while $\beta$-gal staining co-localized with the majority of Aire staining and stained also all of the LacZ positive cells. DAPI was used for nuclear staining. (B) Representative immunohistochemical stainings of Aire and LacZ in Aire+/- mice at embryonic days e14.5 and e15.5. At e14.5 there was a clear signal of Aire but not LacZ, whereas at e15.5 both Aire and LacZ were present albeit always in different cells. (C) Summary of staining for Aire, LacZ, CK10, and involucrin during ontogeny of Aire $+/-$ mice as determined by
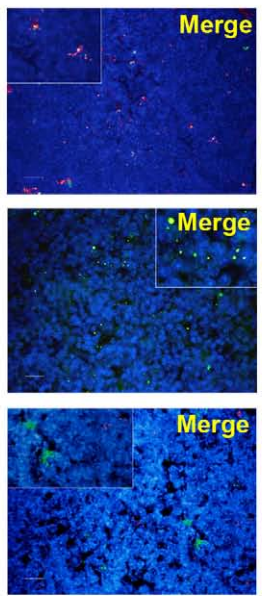

C

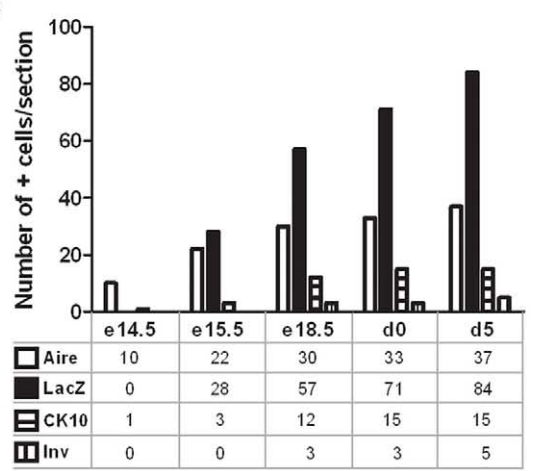

E

\begin{tabular}{c|cc|cc}
\multicolumn{2}{c}{ CK6 } & CK10 & LEKTI & INV \\
\hline Aire & $10-20 \%$ & $10-20 \%$ & $<5 \%$ & $<5 \%$ \\
\hline LacZ & $10-20 \%$ & $10-20 \%$ & $>60 \%$ & $>80 \%$
\end{tabular}

immunohistochemistry. Aire signal was first determined at e14.5, followed by LacZ and CK10 at e15.5, followed in turn by involucrin at e18.5. Shown are values from one representative out of two experiments. (D) Representative samples of co-stainings for LacZ and four epidermal differentiation markers in thymi of 6-week-old Aire+/- mice. (E) Summary of co-stainings for Aire vs. enzymatic LacZ and four epidermal differentiation markers in thymi of 6-week-old Aire+/- mice. The minority of CK6 and CK10 co-localized with Aire or with LacZ. LEKTI and involucrin showed almost no co-staining with Aire, whereas the majority of LEKTI and involucrin positive cells co-localized with LacZ. Values represent the range of at least three experiments, bars correspond to $40 \mu \mathrm{m}$.
II high mTECs (i.e., the population known to express Aire), and against the LacZ- MHC class II low mTECs (i.e., the population known not to express Aire). As expected, Aire was expressed only by the class II high mTECs, with virtually no expression in the class
II low or post-Aire populations (Figure 5B). Rather strikingly, the post-Aire population had also lost almost all of the expression of MHC class II and CD80 and the expression of most of the Aire-dependent TSAs (with the exception of TFF3; Kont et al., 


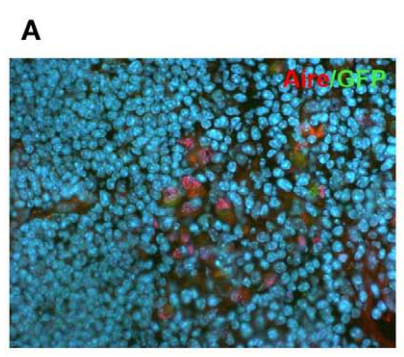

B
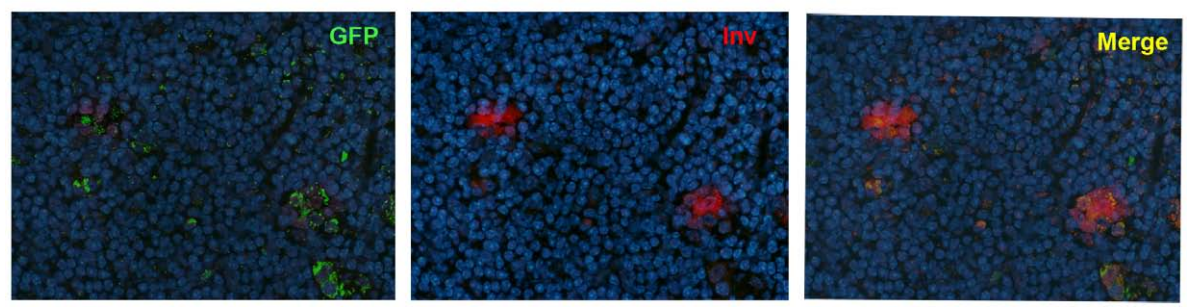

FIGURE 4 | Co-localization of Aire or involucrin with GFP in Adig mice. (A) Thymi from 4-week-old heterozygous Adig mice were stained with Aire antibody and GFP-antibody. The majority of Aire positive cells were also positive for GFP. (B) Thymi from 4-week-old heterozygous Adig mice were stained for involucrin and GFP. Involucrin positive HCs were always positive for GFP. DAPI was used for nuclear staining. Figures are representatives of two independent experiments. See also Figure A5 in Appendix for additional stainings/controls.
2008). Interestingly, the post-Aire population had lost also the expression of Aire-independent TSAs (Derbinski et al., 2005), and had a very low expression level of the Aire-dependent chemokines CCL19 and CCL21 (Laan et al., 2009). The only group of genes showing a preserved or even further increased expression level was the one related to keratinization (Figure 5B), suggesting that the post-Aire stage of mTEC development preferentially preserves the expression pattern commonly associated with the maturation of epidermal epithelium.

We also aimed to purify the HCs by laser microdissection to determine their gene expression profile. However, as HCs contain very few nucleated cells, we were unable to purify RNA even from hundreds of HCs, and thus, we were able to characterize these end-stage mTECs only by the protein expression (see below).

\section{POST-Aire mTECs AND HCs ARE POSTMITOTIC, NON-APOPTOTIC, AND SPECIFICALLY EXPRESS THE PEMPHIGUS VULGARIS-RELATED TSAS DESMOGLEIN-1 AND -3}

As the Aire+ stage of mTECs has been characterized as postmitotic and pro-apoptotic (Gray et al., 2007), we aimed to clarify whether the same stands also for the post-Aire cell populations/HCs. We were unable to see almost any co-staining neither for enzymatic LacZ and a proliferation marker Ki67 nor for LacZ and an apoptosis marker pH2AX (Figure A7 in Appendix). Neither was there any co-staining for the $\mathrm{HCs}$ and the respective proliferation/apoptosis markers (Figure A7 in Appendix). We further looked at the apoptotic status of the Aire positive cells and HCs by using an in situ TUNEL staining together with Aire and involucrin. Similarly to $\mathrm{pH} 2 \mathrm{AX}$, we could not see almost any colocalization of apoptosis and either Aire or involucrin (Figure A8 in Appendix), collectively indicating that at least a subpopulation of Aire + mTECs in addition to remaining non-proliferative also stays non-apoptotic throughout the post-Aire mTEC/HC stages of development.

Because the post-Aire mTECs specifically expressed genes characteristic for keratinocytes, we evaluated whether the epidermal autoantigens are also expressed by this cell population. Thus, we co-stained both post-Aire mTECs and HCs for the pemphigusrelated TSAs desmoglein (DG)-1 and DG-3 (Wada et al., 2011). Both DG-1 and DG-3 were almost exclusively expressed by postAire cells/HCs (Figures 6A,B), indicating that these terminally differentiated mTECs may contribute to tolerance by expressing keratinocyte-related TSAs.

In order to determine whether these keratinocyte-related autoantigens can be presented to the developing thymocytes directly, we co-stained the post-Aire mTECs and HCs with MHC class II as well as CD80. There was a progressive loss from high (Aire+ mTECs) to low (LacZ+ post-Aire mTECs) to virtually negative (HCs) expression (Figure A9 in Appendix) of both markers related to the mTECs properties to directly present the TSAs. However, when the thymi we co-stained for the post-Aire mTECs/HCs and CD11c and LAMP-1, both of the APC markers often localized in conjunction with the post-Aire mTECs as well as HCs (Figures 6A,B). Thus, in agreement with the previous work on end-stage mTEC differentiation (Nishikawa et al., 2010), the postAire cells, and HCs are surrounded by the APCs, being compatible with the idea that the specific TSAs expressed by the terminally differentiated mTECs can be presented to the developing thymocytes by cross-presentation.

\section{DISCUSSION}

The research on HC development and function has been partly limited due to the small size of HCs in the mouse thymus, where, accordingly, their presence and characteristics can be hard to 
A

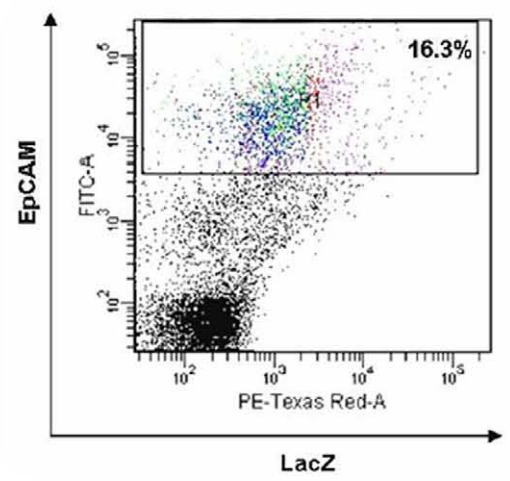

B
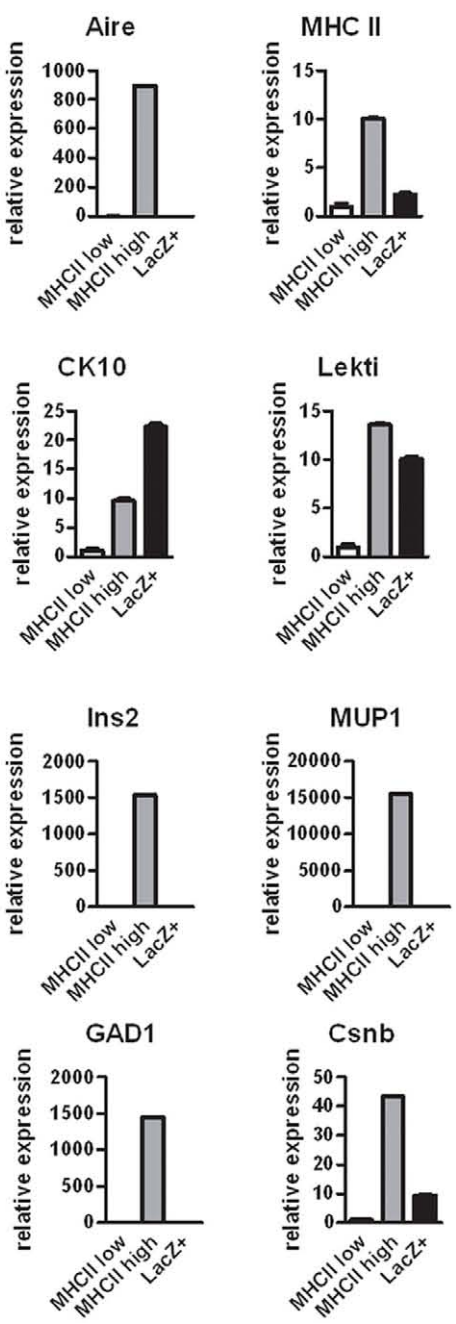

FIGURE 5 | Gene expression profile of the post-Aire mTEC population. (A) Cell sorting strategy to obtain the EpCAM+ ${ }^{+} \mathrm{LaCZ}^{-} \mathrm{MHC} \mathrm{C}^{\circ}$ (P3),

$\mathrm{EpCAM}^{+} \mathrm{LacZ}^{-} \mathrm{MHC}^{\text {hi }}(\mathrm{P} 2)$, and $\mathrm{EpCAM}^{+} \mathrm{LacZ}^{+}$(P4) mTECs from the pooled thymi of Aire+/- mice. (B) Gene expression of selected epidermal markers (CK10, LEKTI, CK2-8), Aire-dependent TSAs (Ins2, MUP1, SPT1, TFF3),

Aire-independent TSAs (GAD1, Csnb) and thymic chemokines (CCL19, CCL21)
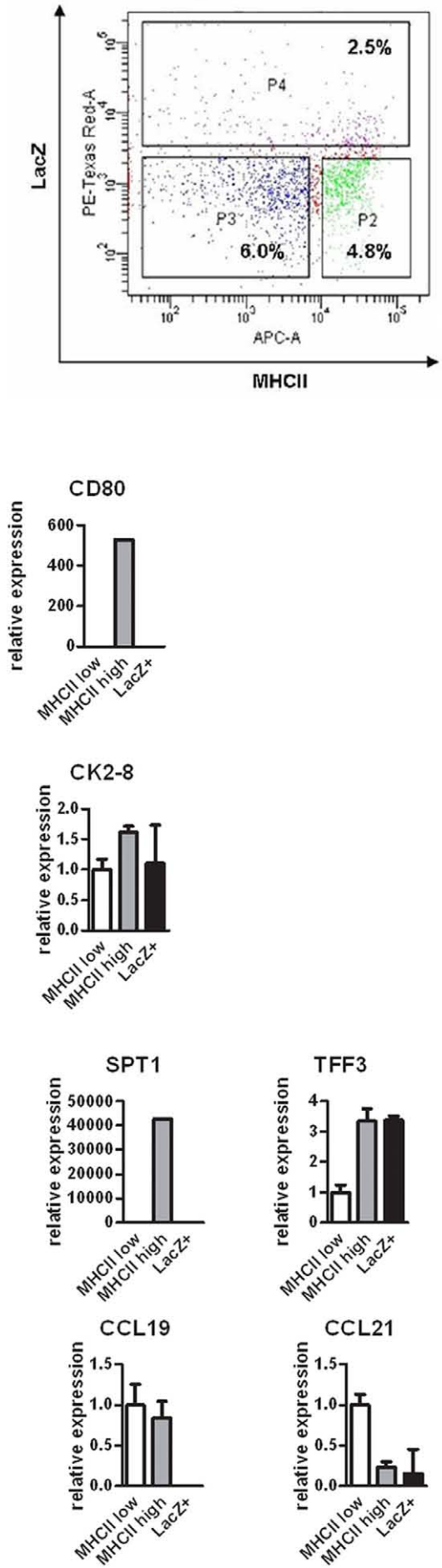

in the post-Aire EpCAM+ $\mathrm{LacZ}^{+}$mTECs compared with the $\mathrm{EpCAM}^{+} \mathrm{LacZ}^{-} \mathrm{MHCl}^{\text {lo }}$ and $\mathrm{EpCAM}^{+} \mathrm{LacZ}^{-} \mathrm{MHCl}^{\text {hi }}$ mTECs as measured by qPCR. The post-Aire population was characterized by a marked decrease in most of the Aire-dependent and Aire-independent genes measured, with the exception of the genes characteristic for epidermal differentiation. Data are mean \pm SEM of triplicate measurement of pooled samples from 15 mice. 
A

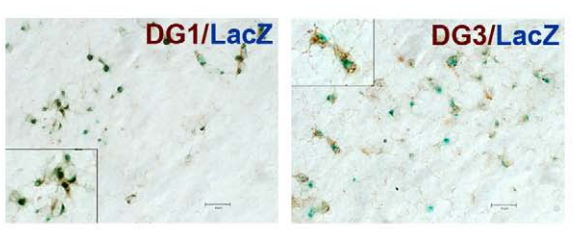

B
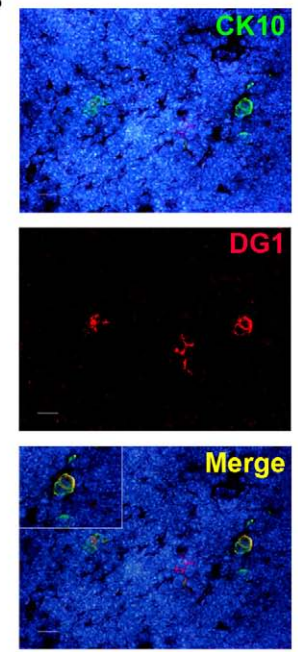
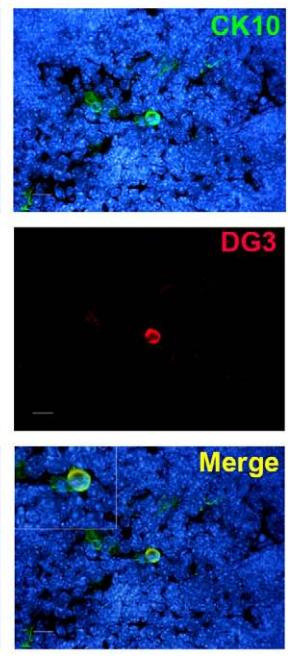
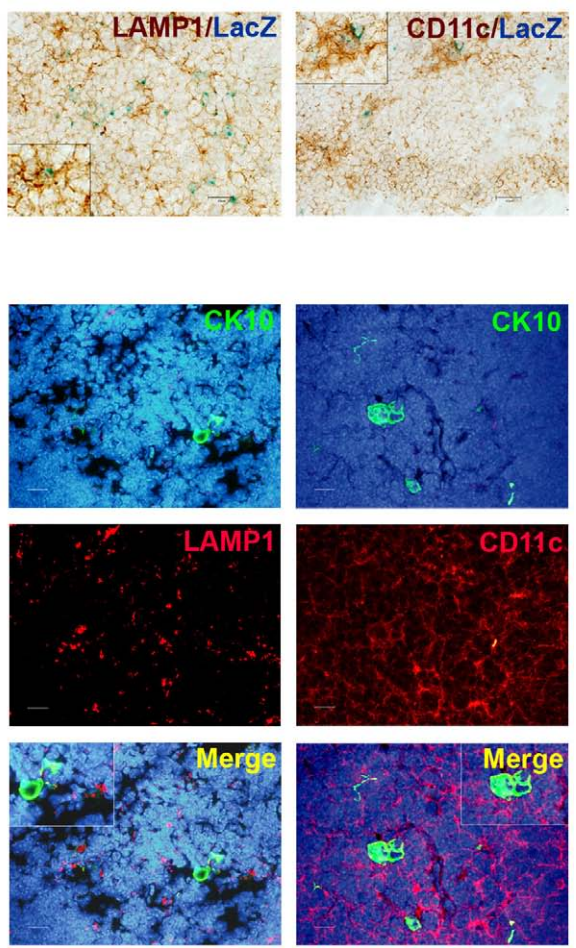

FIGURE 6 | Co-localization of post-Aire mTECs and HCs with epidermal autoantigens and APC markers. (A) Thymi from 4- to 6-week-old Aire+/- mice were stained for LacZ, DG-1, DG-3, LAMP-1, and $\mathrm{CD} 11 \mathrm{c}$. There was a clear co-localization of the $(\mathrm{LacZ}+)$ post-Aire cell population and the autoantigens DG-1 and DG-3. Also, the post-Aire cells were often surrounded by the LAMP-1+ and CD11c+ APCs. (B) Thymi evaluate based on morphology alone. However, as HCs stain for markers of epidermal differentiation both in mice and humans (Bodey et al., 2000; Hale and Markert, 2004), their resemblance to differentiated keratinocytes has been suggested. We show in this study that mouse HCs stain positive for all four epidermal markers used. Whereas involucrin and LEKTI stained only the uppermost layers of skin and were specific for the HCs in thymus, the expression pattern for CK6 and CK10 was broader both in skin as well as thymus where, in addition to the HCs, also several individual mTECs were positively stained. Altogether, assuming that the different developmental stages of mTECs start from single cells to outer layers of HCs to inner layers of HCs, the data clearly support the analogy of end-stage mTEC and keratinocyte development and points out the similarities between the HCs and the granular (outer part of the HC) as well as corneal (inner part of the HC) stages of keratinocytes. The similarities between keratinocyte maturation and $\mathrm{HC}$ development is also supported by gradual expression pattern of keratinocyte maturation markers in thymus ontogeny, where CK10 expression is followed by the one of involucrin. However, as this study focuses on a very limited number of genes/proteins only, a detailed sequential expression pattern of many other genes/proteins may clearly be distinct, and requires more complex evaluation in order to define the true extent of analogy between these two cell types. from 5- to 6-week-old Aire+/- mice were stained for HCs with CK10 and DG-1, DG-3, LAMP-1, and CD11c. There was a clear co-localization of HCs and the epidermal autoantigens DG-1 and DG-3. Also, the HCs were often surrounded by the LAMP-1+ and CD11c+ APCs. In (B) DAPI was used for nuclear staining. Figures are representatives of at least three experiments, bars correspond to $40 \mu \mathrm{m}$.

The role of Aire in regulating mTEC differentiation has been recently indicated in multiple studies (Hale and Markert, 2004; Gillard and Farr, 2005; Gillard et al., 2007; Gray et al., 2007). This study shows a reduced expression of all four different epidermal markers in the Aire KO mice including the extensively expressed CK6 and CK10. However, although reduced in numbers, some $\mathrm{HCs}$ are clearly present even in the Aire KO mice. Thus, while the data on one hand is suggesting a fairly broad defect in mTEC differentiation in the Aire $\mathrm{KO}$ mice, it also indicates that Aire is not absolutely necessary for the development of the final stages of mTEC maturation. As, under proper stimulation by TNFRSF, the numbers of HCs are induced nearly to the normal levels seen in WT thymi, the data collectively suggest a central but dispensable role for Aire in mTEC differentiation/HC development.

Despite of the suggested central role for Aire in controlling $\mathrm{HC}$ development, there had not been any direct evidence that Aire+ mTECs and HCs are forming the same cell lineage. On the contrary, both previous (White et al., 2010), as well the present work shows virtually no co-localization for Aire protein and the HCs. Thus, we hypothesized that during the 2- to 3-week lifecycle of a single mTEC (Gabler et al., 2007), Aire is expressed only within a limited time and, due to its reportedly short half-life, is already degraded once HCs appear. To prove this, we compared the expression of the Aire protein with the one of LacZ staining in the 
heterozygous Aire-LacZ mouse in different time-points of development and also analyzed the expression pattern of GFP and HCs in the Adig mouse. The obtained expression pattern collectively suggests the following: (1) most of the HCs are directly derived from the Aire+ cells as the majority of HCs in the Aire-LacZ mouse are also positive for LacZ, whereas in the Adig mouse the HCs are also positive for GFP. (2) Aire is only expressed once in a given $\mathrm{mTEC}$, as there is no co-localization of Aire protein and LacZ staining. (3) In a given mTEC, Aire is expressed during a limited time with the possible maximal time-frame of 1-2 days, as the signal for Aire protein appears between e13.5 and e14.5, and has disappeared in the same cells by e15.5, when the first post-Aire (i.e., LacZ+) cells appear.

However, although in the two different Aire-reporter mice (Aire-LacZ and Adig) the majority of Aire positive mTECs were also positive for the reporter (65\% in heterozygous Aire-LacZ mice, $84 \%$ in heterozygous Adig mice), there was still a significant proportion of mTECs in both models which did not co-stain. While several technical reasons, such as sensitivity of a particular antibody combination, may easily contribute to this discrepancy, allele-specific expression of Aire (and reporter) in different cells can also be considered. If true, a certain number of Aire-reporterpositive cells seen in the HCs may have in fact been negative for Aire itself. Nevertheless, as at the same time, the development of HCs is clearly Aire-dependent, a major contribution of these relatively rare reporter-positive/Aire-negative cells to $\mathrm{HC}$ development is very unlikely.

As the distinct staining pattern of Aire vs. LacZ allowed us to distinguish and purify the post-Aire cell population, we compared the gene expression profile of the Aire-, Aire+, and post-Aire mTECs in order to see whether the unique features of Aire positive mTECs also remain during the post-Aire stage of development. Although this study covered only a fraction of genes, it seems quite clear that the "opened" pattern of gene expression is characteristic to the MHC class II high mTECs only and, during further development, the expression pattern of mTECs becomes more restricted and favors the expression of keratinocyte-related genes and proteins, including the known keratinocyte-related TSAs. Interestingly, the progressive restriction model of mTEC differentiation has been suggested as an alternative for the more wildly accepted model of terminal differentiation (Gillard and Farr, 2005; Matsumoto, 2011). The current data indicates that, although the gene expression pattern becomes more complex during the differentiation up to the Aire+ stages of development, the mTECs at the final stages of differentiation become indeed more restricted in their gene expression pattern. Thus, the current study suggests that the two proposed models can in fact describe the process of mTEC differentiation at different stages of mTEC maturation.

Although Aire has been shown to induce apoptosis in mTECs, we see no signs of apoptosis in the post-Aire mTEC population or in the HCs. Thus, although we cannot exclude that some of the Aire + cells indeed undergo apoptosis and are perhaps quickly cleared by the phagocytes and that some epithelial apoptosis remains undetected by the stainings used in this study, there seems to be a surviving post-Aire mTEC population that, by loosing the nuclei and forming the HCs, rather resembles the keratinization of stratum corneum of the epidermis. However, as in thymus desquamation is not likely to occur, the processes behind cleaving and removing of the HCs remain to be clarified in future studies.

In conclusion, we showed that the short-living Aire+ stage of mTEC development is followed by the Aire- stage of mTEC development, followed in turn by the loss of nuclei, and the formation of HCs. Whereas the unique abilities of Aire+ mTECs to express and present a variety of TSAs are lost during their further maturation, the post-Aire stages are characterized by the specific expression of keratinocyte-related genes, including the pemphigus-related autoantigens DG-1 and DG-3. Finally, we showed that, although clearly dependent on Aire expression, the final stages of mTEC maturation can be induced by TNFRSF ligands even in the absence of Aire.

\section{MATERIALS AND METHODS \\ MICE}

Mice deficient for Aire gene were generated at the Walter and Eliza Hall Institute (Melbourne, Australia) by a homologous recombination of targeting vectors in mouse C57BL/6 embryonic stem cells (Hubert et al., 2009). Insertion of the Aire-targeting vector disrupted exon 8 and brought the LacZ reporter gene under the control of the endogenous Aire promoter, creating an AireLacZ fusion. The Aire knockout mutation was backcrossed onto $\mathrm{BALB} / \mathrm{c}$, for 10 generations and intercrossed once for analysis.

As an alternative model for tracing the post-Aire cells, the Airedriven IGRP-GFP (Adig) mouse was used (Gardner et al., 2008). In this transgenic mouse, Aire drives the expression of GFP fused to IGRP. The Adig mouse has been generated and characterized at the Diabetes Center, University of California San Francisco, USA and was a kind gift from Prof. Mark S. Anderson. Wildtype (WT), Aire-LacZ heterozygous (Aire+/-), and Aire-LacZ knockout (Aire KO) mice were used on $\mathrm{C} 57 \mathrm{BL} / 6$ and $\mathrm{BALB} / \mathrm{c}$ backgrounds. The Adig mice on $\mathrm{BALB} / \mathrm{c}$ background was used in heterozygosity only. All mice were bred and maintained at the mouse facility of the Institute of Molecular and Cell Biology, Tartu University. Permission to carry out these animal experiments was issued by the Estonian State Committee for Licensing of Animal Experiments. Unless otherwise indicated, 4- to 8-week-old mice were used primarily on C57BL/6 background. The crucial findings on identification and characterization of the post-Aire cell population was performed both in Aire+/-mice on C57BL/6 background as well as on BALB/c background.

\section{ANTIBODIES}

The following primary Abs were used: anti-Aire rabbit polyclonal, anti-LEKTI rabbit polyclonal antibody, anti-CK10 goat polyclonal, anti-desmoglein-1 goat polyclonal, anti-desmoglein 3 goat polyclonal antibody were all from Santa Cruz Biotechnology (Santa Cruz, USA); anti-CK6 rabbit polyclonal, antiCK10 rabbit polyclonal, anti-involucrin rabbit polyclonal, antiKi67 rabbit polyclonal, anti-phospo-H2AX rabbit polyclonal, anti-MHCII rat monoclonal, anti-LAMP-1 rat monoclonal, antiphospho-H2AX mouse monoclonal, anti- $\beta$-galactosidase goat polyclonal, anti-GFP chicken polyclonal were all from Abcam (Fremont, USA); anti-MHCII-APC and anti-CD11c-Cy5.5 were both from Miltenyi Biotec (Bergisch Gladbach, Germany); anti- $\beta$ galactosidase mouse monoclonal from Promega (Madison, USA). 
The anti-EpCAM antibody and anti-EpCAM-FITC was generated in-house in the Molecular Pathology Group, Tartu University from G8.8 hybridoma cell line, which was obtained from Developmental Studies Hybridoma Bank, University of Iowa, USA. The rat antiAire $\mathrm{mAb}$ was produced in the Monoclonal Antibody Facility at the Walter and Eliza Hall Institute of Medical Research (Melbourne, Australia; Hubert et al., 2008).

The following horseradish peroxidase (HRP) labeled secondary antibodies were used for immunohistochemistry: anti-rabbit swine polyclonal, anti-rat goat polyclonal, and anti-goat donkey polyclonal (all from Santa Cruz Biotechnology). For immunofluorescence anti-rat goat polyclonal antibody conjugated with Alexa Fluor 488, anti-rat goat polyclonal antibody conjugated with Alexa Fluor 594, anti-rabbit goat polyclonal antibody conjugated with Alexa Fluor 488, anti-rabbit goat polyclonal antibody conjugated with Alexa Fluor 594, anti-goat donkey polyclonal antibody conjugated with Alexa Fluor 594, and anti-rabbit chicken polyclonal antibody conjugated with Alexa Fluor 488 (all from Life Technologies, Carlsbad, USA) were used.

\section{TISSUE STAININGS}

Tissues from WT, Aire+/-, and Aire KO mice were fixed in 10\% neutral buffered formalin, and then processed into paraffin blocks using standard protocols. Two sections from each sample were stained using the hematoxylin-eosin staining to observe the overall structure, number, and shape of the HCs. Simultaneously, some thymus tissues were snap-frozen in TissueTek (Vogel, Germany) and $5 \mu \mathrm{m}$ frozen sections were made and stored at $-80^{\circ} \mathrm{C}$ for later use.

For all immunohistochemical or immunofluorescent stainings one stained section on a glass was paralleled with one negative control section on the same glass, which went through exactly the same procedures but instead of the primary antibody was incubated with phosphate buffer solution (PBS) only. On the glasses used for analysis, the negative controls showed virtually no signal.

For immunohistochemistry, the immunoperoxidase assays were performed using standard techniques on $4 \%$ formaldehydefixed frozen sections or chamber slides. Endogenous peroxidase was blocked with $3 \% \mathrm{H}_{2} \mathrm{O}_{2}$. Non-specific protein binding was blocked by incubation with $5 \%$ normal swine or goat serum. Sections or cells were sequentially incubated with primary antibodies for $60 \mathrm{~min}$ at $37^{\circ} \mathrm{C}$, swine anti-rabbit secondary antibodies, or goat anti-rat secondary antibodies for $60 \mathrm{~min}$ at $37^{\circ} \mathrm{C}$, with intervening PBS washes. After a complete wash in PBS, the slides were developed in $0.05 \%$ freshly prepared $3,3^{\prime}$-diaminobenzidine solution with $0.03 \%$ hydrogen peroxide for $5 \mathrm{~min}$, and then counterstained with hematoxylin, dehydrated, air-dried, and permanently mounted. For frozen sections, the optimum dilution of each antibody was first determined by serial dilutions on WT mouse thymus sections. Normal swine or goat serum was used to substitute for the primary antibody to serve as a negative control. No specific immunoreactivity was detected in these tissue sections. The images were captured using an Olympus IX70 inverted microscope equipped with WLSM PlanApo $20 \times$ or $40 \times$ water immersion objective and Olympus DP70 CCD camera (Olympus Corp., Tokyo, Japan).
For histochemical $\beta$-galactosidase staining, the frozen sections were fixed in ice-cold $4 \%$ formaldehyde for $10 \mathrm{~min}$, then washed in $0.1 \mathrm{M}$ cold PBS with $1 \mathrm{mM} \mathrm{MgCl}_{2}$ for $20 \mathrm{~min}$, and stained with $0.1 \mathrm{M}$ PBS containing $0.2 \%$ X-gal, $5 \mathrm{mM} \mathrm{K}_{4} \mathrm{Fe}(\mathrm{CN})_{6}, 5 \mathrm{mM}$ $\mathrm{K}_{3} \mathrm{Fe}(\mathrm{CN})_{6}$ in the dark at $4^{\circ} \mathrm{C}$ overnight. After a wash with $1 \mathrm{M}$ PBS, co-immunostaining with different markers was carried out as described above.

Immunofluorescence was performed on frozen sections fixed with $4 \%$ formaldehyde. The tissues were blocked in $1 \%$ normal goat serum for $10 \mathrm{~min}$ at room temperature, and then incubated with indicated primary antibody for $60 \mathrm{~min}$ at room temperature. After wash in PBS, the cells were incubated with Alexa Fluor 488 or Alexa Fluor 594 labeled secondary antibody (1:2000) for $60 \mathrm{~min}$ at room temperature.

For fluorescent enzymatic LacZ staining the frozen sections were stained with the ImaGene Red C12RG $\beta$-galactosidase substrate (Invitrogen, Leiden, the Netherlands) as recommended by the manufacturer and analyzed as described above.

TUNEL staining was performed on frozen thymic sections by using TACS ${ }^{\circledR}$ 2TdT In situ Apoptosis Detection Kit (Trevigen Inc., Gaithersburg, USA) as recommended by the manufacturer.

\section{QUANTITATIVE REAL-TIME PCR}

RNA was extracted from snap-frozen mouse tissues using TRIzol (Invitrogen/Life Technologies) and reverse-transcribed to cDNA using the SuperScript III Reverse Transcriptase (Invitrogen/Life Technologies). qPCR was performed with the Applied Biosystems Prism 7900 SDS instrument using a qPCR SYBR Green Core Kit (Eurogentec) according to the manufacturer's instructions.

The amplification program included an initial denaturation step at $95^{\circ} \mathrm{C}$ for $10 \mathrm{~min}$, followed by denaturation at $95^{\circ} \mathrm{C}$ for $15 \mathrm{~s}$, and annealing and extension at $60^{\circ} \mathrm{C}$ for $1 \mathrm{~min}$ for 45 cycles. SYBR Green fluorescence was measured after each extension step, and the specificity of amplification was evaluated by a melting curve analysis. Every sample was run in three parallel reactions. Primers used to amplify specific gene products from murine cDNA were as follows: Aire (F: 5' -TCCTCAATGAGCACTCATTTGAC3', R: 5'-CCACCTGTCATCAGGAAGAG-3'), CK6 (F: 5' CAAACTCACATCTCAGACAC-3' ${ }^{\prime}$ R: $5^{\prime}$-GCAGCTCCTCATATTT AGTC-3'), CK10 (F: 5'-GTTCAATCAGAAGAGCAAGGA-3', R: 5'-GTAGTTCAATCTCCAGACCC-3'), LEKTI (F: 5' -CGAAGGCT AAGGATGAATGTG-3', R: 5'-GCAGTAGTTCTTTACACATGA$3^{\prime}$ ), Involucrin (F: 5'-GTGAGTTTGTTTGGTCTACAG-3', R: 5' GAAAGCCCTTCTCTTGAATCTC-3'), TSLP (F: 5'-GACAGCAT GGTTCTTCTCAG-3'， R: 5'-CTGGAGATTGCATGAAGG-3'), CD80 (F: 5' - GTCCATCAAAGCTGACTTCTC-3', R: 5'-ATGC CAGGTAATTCTCTTCCA-3'), MHCII (F: $5^{\prime}$-CTCAGAAATAGCA AGTCAGTC-3', R: 5'-AATCTCAGGTTCCCAGTG-3'), Insulin2 (F: $5^{\prime}$-CAAGTGGCACAACTGGAC-3' ${ }^{\prime}$ R: $5^{\prime}$-CAGCACTGATCTA CAATG-3' ${ }^{\prime}$ ), TFF3 (F: 5' -TACGTTGGCCTGTCTCCAAG-3', R: $5^{\prime}$ CAGGGCACATTTGGGATACT-3'), MUP1 (F: $5^{\prime}$-TCTGTGACGT ATGATGGATTCAA-3'， R: 5'-TCTGGTTCTCGGCCATAGAG3'), Spt1 (F: 5'-AACTTCTGGAACTGCTGATTCTG-3', R: 5'GAGGCCTCATTAGCAGTGTTG-3'), GAD1 (F: 5' -ATAGAAAGG GCCAATTCAGTC-3', R: 5'-TGCATCCTTGGAGTATACCC-3'), Csnb (F: 5'-GGCACAGGTTGTTCAGGCTT-3', R: 5' -AAGGAAG GGTGCTACTTGCTG-3'), Dsg1 (F: 5' -CTTAGGATTGGTTCCAT 
TCCTG-3', R: 5'-CCATTAGTTCAAATCCTGTGGT-3'), Dsg3 (F: $5^{\prime}$-TTCGGAAGGTACAATTCATCAG-3' ${ }^{\prime}$ R: $5^{\prime}$-TGCAAACTTCAG AGCTTTCC-3'), CK2-8 (F: 5'-AGGAGCTCATTCCGTAGCTG3', R: 5'-TCTGGGATGCAGAACATGAG-3'), CCL19 (F: 5'CTGCCTCAGATTATCTGCCAT- $3^{\prime}$, R: 5' -CTTCCGCATCATTAG CACCC-3'), CCL21(F: 5'-CCCTGGACCCAAGGCAGT-3', R: 5' AGGCTTAGAGTGCTTCCGGG-3'), $\beta$-2-microglobulin (F: $5^{\prime}$ TGAGACTGATACATACGCCTGCA-3' ${ }^{\prime}$ R: $5^{\prime}$-GATGCTTGATCAC ATGTCTCGATC- $\left.3^{\prime}\right)$. The relative gene expression levels were calculated using the comparative Ct $(\Delta \Delta C \mathrm{t})$ method (according to Applied Biosystems), where the relative expression is calculated as $2^{-\Delta \Delta C t}$, and where $C t$ represents the threshold cycle. $\beta$-2-microglobulin was used as a house-keeping gene for normalization.

\section{EX VIVO STIMULATION OF THYMIC CULTURES}

Whole thymi from 4- to 6-week-old Aire KO mice (or WT controls) were dissected and cultured in a standard cell-culture incubator at the air-liquid interface on the Isopore Membrane Filters (Millipore) in DMEM supplemented with 10\% FCS and antibiotics. The thymi were treated with LIGHT $(50 \mathrm{ng} / \mathrm{ml}+$ polyHis $2.5 \mu \mathrm{g} / \mathrm{ml})$, RANKL $(15 \mathrm{ng} / \mathrm{ml})$, or CD $40 \mathrm{~L}(10 \mathrm{ng} / \mathrm{ml}$, all from $\mathrm{R} \& \mathrm{D}$ ) and collected for 24 or $48 \mathrm{~h}$ later for analysis by qPCR or immunohistochemistry, respectively.

\section{FACS SORTING AND ANALYSIS OF SORTED CELLS}

Fifteen thymi from 6- to 8-week-old heterozygous mice were dissected and collected into ice-cold PBS. GentleMACS C-Tubes (Miltenyi Biotec) were used for mechanical disruption followed by enzymatic digestion in $0.5 \mathrm{mg} / \mathrm{ml}$ dispase/collagenase (Roche) and $5 \mu \mathrm{g} / \mathrm{ml}$ DNase I (AppliChem) in PBS at $37^{\circ} \mathrm{C}$ for $3 \times 30 \mathrm{~min}$ with gentle agitation. After each incubation period, the released cells were counted and, starting from the last fraction, the cells

\section{REFERENCES}

Abramson, J., Giraud, M., Benoist, C., and Mathis, D. (2010). Aire's partners in the molecular control of immunological tolerance. Cell 140, 123-135.

Akiyama, T., Shimo, Y., Yanai, H., Qin, J., Ohshima, D., Maruyama, Y., Asaumi, Y., Kitazawa, J., Takayanagi, H., Penninger, J. M., Matsumoto, M., Nitta, T., Takahama, Y., and Inoue, J. (2008). The tumor necrosis factor family receptors RANK and CD40 cooperatively establish the thymic medullary microenvironment and self-tolerance. Immunity 29, 423-437.

Anderson, M. S., and Su, M. A. (2011). Aire and T cell development. Curr. Opin. Immunol. 23, 198-206.

Anderson, M. S., Venanzi, E. S., Klein, L., Chen, Z., Berzins, S. P., Turley, S. J., Von Boehmer, H., Bronson, R., Dierich, A., Benoist, C., and Mathis, D. (2002). Projection of an immunological self shadow within the thymus by the Aire protein. Science 298, 1395-1401.
Bodey, B., Bodey, B. Jr., Siegel, S. E., and Kaiser, H. E. (2000). Novel insights into the function of the thymic Hassall's bodies. In vivo $14,407-418$.

Derbinski, J., Gabler, J., Brors, B., Tierling, S., Jonnakuty, S., Hergenhahn, M., Peltonen, L., Walter, J., and Kyewski, B. (2005). Promiscuous gene expression in thymic epithelial cells is regulated at multiple levels. J. Exp. Med. 202, 33-45.

Derbinski, J., Schulte, A., Kyewski, B., and Klein, L. (2001). Promiscuous gene expression in medullary thymic epithelial cells mirrors the peripheral self. Nat. Immunol. 2, 1032-1039.

Dooley, J., Erickson, M., and Farr, A. G. (2008). Alterations of the medullary epithelial compartment in the Aire-deficient thymus: implications for programs of thymic epithelial differentiation. J. Immunol. 181, 5225-5232.

Gabler, J., Arnold, J., and Kyewski, B. (2007). Promiscuous gene expression and the developmental dynamics of medullary thymic epithelial cells. Eur. J. Immunol. 37, 3363-3372.

were pooled to gain $200 \times 10^{6}$ cells. In this population, a negative selection was performed with CD45 microbeads and AutoMACS (Miltenyi Biotec) to obtain the CD45- cells. To get the populations of diverse mTECs, the $\mathrm{CD} 45^{-}$thymic stromal cells were stained with LacZ (X-gal, ImaGene Red C12RG $\beta$-galactosidase substrate, Invitrogen), anti-EpCAM monoclonal $\mathrm{Ab}$, and antiMHCII monoclonal Ab (Miltenyi Biotec), followed by FACS sorting with a FACS Vantage (BD). According to EpCAM, LacZ, and MHCII expression, the mTEC fractions were further divided into the $\mathrm{EpCAM}^{+} \mathrm{LacZ}^{-} \mathrm{MHCII}^{\mathrm{lo}}, \mathrm{EpCAM}^{+} \mathrm{LacZ}^{-} \mathrm{MHCII}{ }^{\mathrm{hi}}$, and EpCAM $^{+}$LacZ $^{+}$mTECs. RNA was purified by an RNeasy Micro Kit (Qiagen) followed by reverse transcription and $\mathrm{qPCR}$ as described above.

\section{LASER MICRODISSECTION}

Ten micrometer thick frozen sections from WT thymi were placed on PALM membrane slides, stained with CK10 antibody to visualize the HCs, and microdissected with Zeiss Palm MicroBeam. Cellular material from $200 \mathrm{HCs}$ was collected and the RNA purified, cDNA reverse-transcribed and qPCR performed as described above.

\section{ACKNOWLEDGMENTS}

We thank Ms. Maire Pihlap (Tartu University) for technical assistance as well as Prof. Mark Anderson (University of California) and Mr. Felix Klug (German Cancer Research Center) for kind help Re Adig mice. This work was supported by the Estonian Science Foundation Grants 8710, 8358, 8169 (Martti Laan, Kai Kisand, Pärt Peterson); European Regional Development Fund and Archimedes Foundation (Xiaoping Wang, Martti Laan, Kai Kisand, Pärt Peterson); Estonian targeted financiation grant SF0180021s07 (Pärt Peterson); NHMRC fellowship 461204 (H.H.S.); and NHMRC program grants 257501, 264573, 406700, 454465 (H.H.S.).

Gallegos, A. M., and Bevan, M. J. (2006). Central tolerance: good but imperfect. Immunol. Rev. 209, 290-296.

Gardner, J. M., Devoss, J. J., Friedman, R. S., Wong, D. J., Tan, Y. X., Zhou, X., Johannes, K. P., Su, M. A., Chang, H. Y., Krummel, M. F., and Anderson, M. S. (2008). Deletional tolerance mediated by extrathymic Aire-expressing cells. Science 321, 843-847.

Gillard, G. O., Dooley, J., Erickson, M., Peltonen, L., and Farr, A. G. (2007). Aire-dependent alterations in medullary thymic epithelium indicate a role for Aire in thymic epithelial differentiation. $J$. Immunol. 178, 3007-3015.

Gillard, G. O., and Farr, A. G. (2005). Contrasting models of promiscuous gene expression by thymic epithelium. J. Exp. Med. 202, 15-19.

Gray, D., Abramson, J., Benoist, C., and Mathis, D. (2007). Proliferative arrest and rapid turnover of thymic epithelial cells expressing Aire. J. Exp. Med. 204, 2521-2528.
Hale, L. P., and Markert, M. L. (2004). Corticosteroids regulate epithelial cell differentiation and Hassall body formation in the human thymus. J. Immunol. 172, 617-624.

Hubert, F. X., Kinkel, S. A., Crewther, P. E., Cannon, P. Z., Webster, K. E., Link, M., Uibo, R., O’bryan, M. K., Meager, A., Forehan, S. P., Smyth, G. K., Mittaz, L., Antonarakis, S. E., Peterson, P., Heath, W. R., and Scott, H. S. (2009). Aire-deficient C57BL/6 mice mimicking the common human 13-base pair deletion mutation present with only a mild autoimmune phenotype. J. Immunol. 182, 3902-3918.

Hubert, F. X., Kinkel, S. A., Webster, K. E., Cannon, P., Crewther, P. E., Proeitto, A. I., Wu, L., Heath, W. R., and Scott, H. S. (2008). A specific anti-Aire antibody reveals Aire expression is restricted to medullary thymic epithelial cells and not expressed in periphery. J. Immunol. 180, 3824-3832. 
Kekalainen, E., Tuovinen, H., Joensuu, J., Gylling, M., Franssila, R., Pontynen, N., Talvensaari, K., Perheentupa, J., Miettinen, A., and Arstila, T. P. (2007). A defect of regulatory $\mathrm{T}$ cells in patients with autoimmune polyendocrinopathycandidiasis-ectodermal dystrophy. J. Immunol. 178, 1208-1215.

Kont, V., Laan, M., Kisand, K., Merits, A., Scott, H. S., and Peterson, P. (2008). Modulation of Aire regulates the expression of tissue-restricted antigens. Mol. Immunol. 45, 25-33.

Kyewski, B., and Derbinski, J. (2004). Self-representation in the thymus: an extended view. Nat. Rev. Immunol. 4, 688-698.

Laan, M., Kisand, K., Kont, V., Moll, K., Tserel, L., Scott, H. S., and Peterson, P. (2009). Autoimmune regulator deficiency results in decreased expression of CCR4 and CCR7 ligands and in delayed migration of CD4+ thymocytes. J. Immunol. 183, 7682-7691.

Li, J., Li, Y., Yao, J. Y., Jin, R., Zhu, M. Z., Qian, X. P., Zhang, J., Fu, Y. X., Wu, L., Zhang, Y., and Chen, W. F. (2007). Developmental pathway of CD4+CD8- medullary thymocytes during mouse ontogeny and its defect in Aire-/- mice. Proc. Natl. Acad. Sci. U.S.A. 104, 18175-18180.

Liston, A., Lesage, S., Wilson, J., Peltonen, L., and Goodnow, C. C. (2003). Aire regulates negative selection of organ-specific T cells. Nat. Immunol. 4, 350-354.

Matsumoto, M. (2011). Contrasting models for the roles of Aire in the differentiation program of epithelial cells in the thymic medulla. Eur. J. Immunol. 41, 12-17.
Milicevic, Z., Milicevic, N. M., Laan, M., Peterson, P., Kisand, K., Scott, H. S., and Westermann, J. (2010). Ultrastructure of medullary thymic epithelial cells of autoimmune regulator (Aire)-deficient mice. Immunol. Cell Biol. 88, 50-56.

Nishikawa, Y., Hirota, F., Yano, M., Kitajima, H., Miyazaki, J., Kawamoto, H., Mouri, Y., and Matsumoto, M. (2010). Biphasic Aire expression in early embryos and in medullary thymic epithelial cells before end-stage terminal differentiation. J. Exp. Med. 207, 963-971.

Nuber, U. A., Schafer, S., Stehr, S., Rackwitz, H. R., and Franke, W. W. (1996). Patterns of desmocollin synthesis in human epithelia: immunolocalization of desmocollins 1 and 3 in special epithelia and in cultured cells. Eur. J. Cell Biol. 71, 1-13.

Pereira, C., Maamar-Tayeb, M., Burke, A., Perez-Polo, R., Herndon, D. N., and Jeschke, M. G. (2006). Immunohistochemical staining of transgenic beta-galactosidase in burned skin is a better indicator of transfection efficiency than histochemical techniques. J. Immunol. Methods 315, 75-79.

Peterson, P., Org, T., and Rebane, A. (2008). Transcriptional regulation by AIRE: molecular mechanisms of central tolerance. Nat. Rev. Immunol. 8, 948-957.

Peterson, P., and Peltonen, L. (2005). Autoimmune polyendocrinopathy syndrome type 1 (APS1) and AIRE gene: new views on molecular basis of autoimmunity. J. Autoimmun. 25(Suppl.), 49-55.
Pomie, C., Vicente, R., Vuddamalay, Y. Lundgren, B. A., Van Der Hoek, M., Enault, G., Kagan, J., Fazilleau, N., Scott, H. S., Romagnoli, P., and Van Meerwijk, J. P. (2011). Autoimmune regulator (AIRE)-deficient CD8+CD28low regulatory $\mathrm{T}$ lymphocytes fail to control experimenta colitis. Proc. Natl. Acad. Sci. U.S.A. 108, 12437-12442.

Rossi, S. W., Kim, M. Y., Leibbrandt, A., Parnell, S. M., Jenkinson, W. E., Glanville, S. H., Mcconnell, F. M., Scott, H. S., Penninger, J. M., Jenkinson, E. J., Lane, P. J., and Anderson, G. (2007). RANK signals from $\mathrm{CD} 4(+) 3(-)$ inducer cells regulate development of Aireexpressing epithelial cells in the thymic medulla. J. Exp. Med. 204 1267-1272.

Wada, N., Nishifuji, K., Yamada, T. Kudoh, J., Shimizu, N., Matsumoto, M., Peltonen, L., Nagafuchi, S., and Amagai, M. (2011). Aire-dependent thymic expression of desmoglein 3 , the autoantigen in pemphigus vulgaris, and its role in T-cell tolerance. J. Invest. Dermatol. 131, 410-417.

Watanabe, N., Wang, Y. H., Lee, H. K., Ito, T., Wang, Y. H., Cao, W., and Liu, Y. J. (2005). Hassall's corpuscles instruct dendritic cells to induce $\mathrm{CD} 4+\mathrm{CD} 25+$ regulatory $\mathrm{T}$ cells in human thymus. Nature 436, 1181-1185.

White, A. J., Nakamura, K., Jenkinson, W. E., Saini, M., Sinclair, C., Seddon, B., Narendran, P., Pfeffer, K., Nitta, T., Takahama, Y., Caamano, J. H., Lane, P. J., Jenkinson, E. J., and Anderson, G. (2010). Lymphotoxin signals from positively selected thymocytes regulate the terminal differentiation of medullary thymic epithelial cells. J. Immunol. 185, 4769-4776.

Yano, M., Kuroda, N., Han, H., MeguroHorike, M., Nishikawa, Y., Kiyonari, H., Maemura, K., Yanagawa, Y., Obata, K., Takahashi, S., Ikawa, T., Satoh, R., Kawamoto, H., Mouri, Y., and Matsumoto, M. (2008). Aire controls the differentiation program of thymic epithelial cells in the medulla for the establishment of self-tolerance. J. Exp. Med. 205, 2827-2838.

Conflict of Interest Statement: The authors declare that the research was conducted in the absence of any commercial or financial relationships that could be construed as a potential conflict of interest.

Received: 24 August 2011; accepted: 02 February 2012; published online: 05 March 2012.

Citation: Wang X, Laan M, Bichele $R$, Kisand K, Scott HS and Peterson $P$ (2012) Post-Aire maturation of thymic medullary epithelial cells involves selective expression of keratinocyte-specific autoantigens. Front. Immun. 3:19. doi: 10.3389/fimmu.2012.00019

This article was submitted to Frontiers in Immunological Tolerance, a specialty of Frontiers in Immunology.

Copyright (C) 2012 Wang, Laan, Bichele, Kisand, Scott and Peterson. This is an open-access article distributed under the terms of the Creative Commons Attribution Non Commercial License, which permits non-commercial use, distribution, and reproduction in other forums, provided the original authors and source are credited. 


\section{APPENDIX}

\section{A}
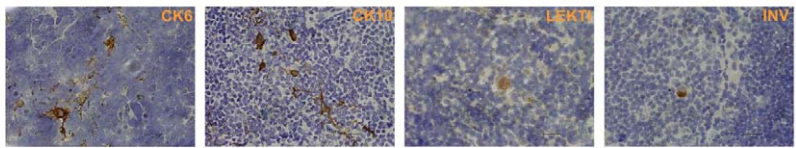

B

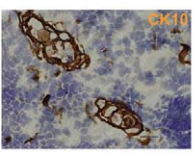

FIGURE A1 | Epidermal differentiation markers in rat and human thymi. (A) Similarly to the stainings in mice, CK6, and CK10 showed a rather broad expression pattern in rat thymi that was localized both in $\mathrm{HCs}$ as well as single mTECs, whereas LEKTI and involucrin stainings were specific for HCs only.
(B) In human thymus, anti-CK10 showed a rather broad expression pattern both in HCs and mTECs, whereas the other antibodies used did not cross-react with human tissue (not shown). Shown are representatives of at least two samples. Bars correspond to $40 \mu \mathrm{m}$
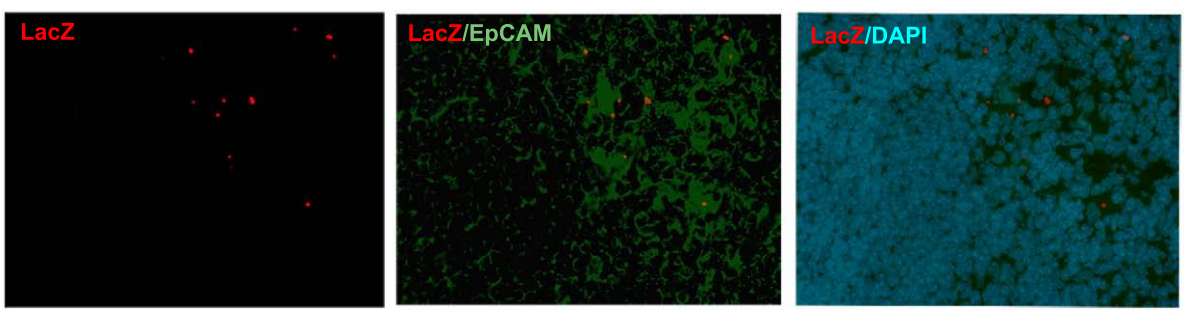

FIGURE A2 | Validation of the fluorescent substrate-based LacZ staining. In the thymi from heterozygous Aire-LacZ mice the speckled staining located in the medullary areas and in the EpCAM positive cells. Shown are representatives of two samples.
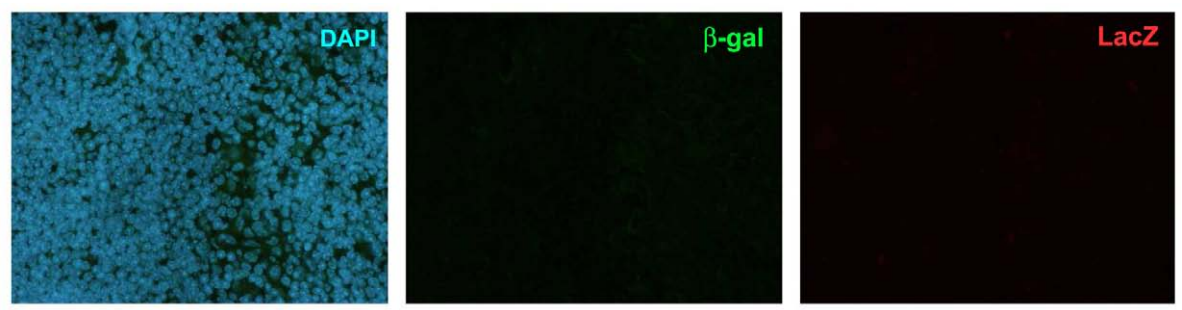

FIGURE A3 | Validation of the $\boldsymbol{\beta}$-gal antibody and enzymatic LacZ kit. There was no staining of neither $\beta$-gal nor enzymatic LacZ in the WT mouse. Shown are representatives of two experiments. 

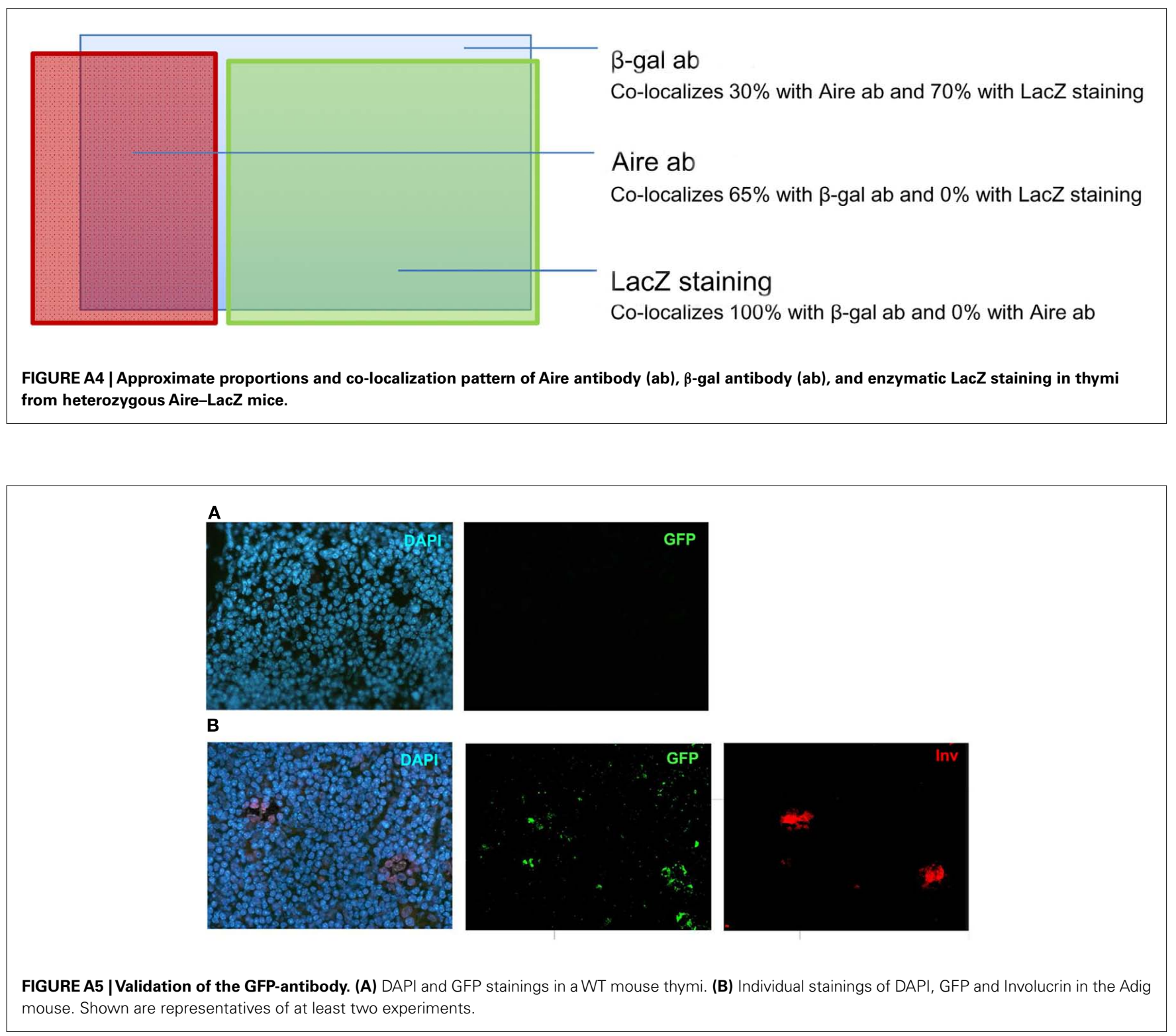

mouse. Shown are representatives of at least two experiments.
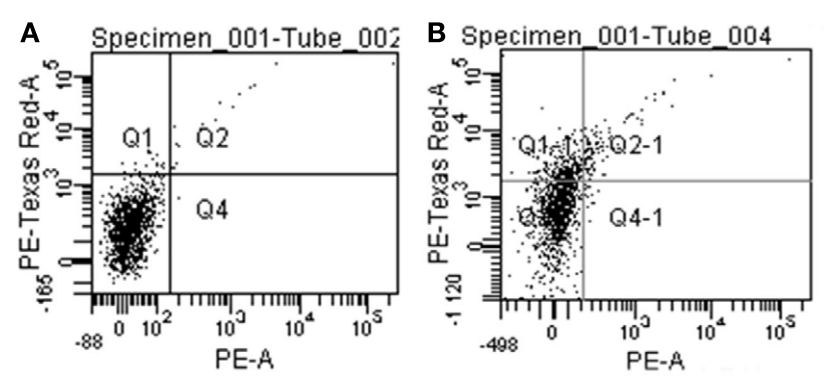

FIGURE A6 | Negative control (A) and stained sample (B) of CD45-depleted thymic stromal cells from heterozygous Aire-LacZ mice. The specific signal appears in the PE-Texas Red-A channel shown on the $Y$-axis. 

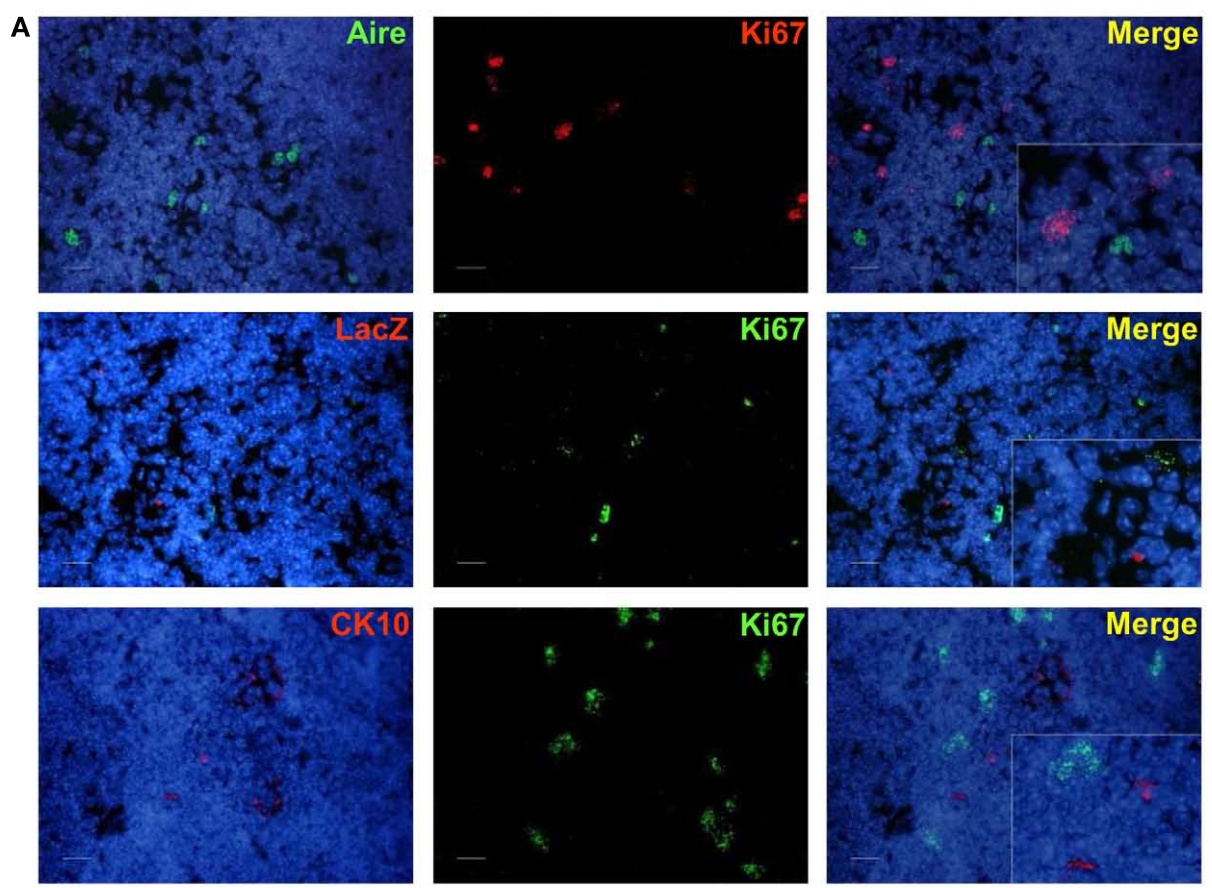

B
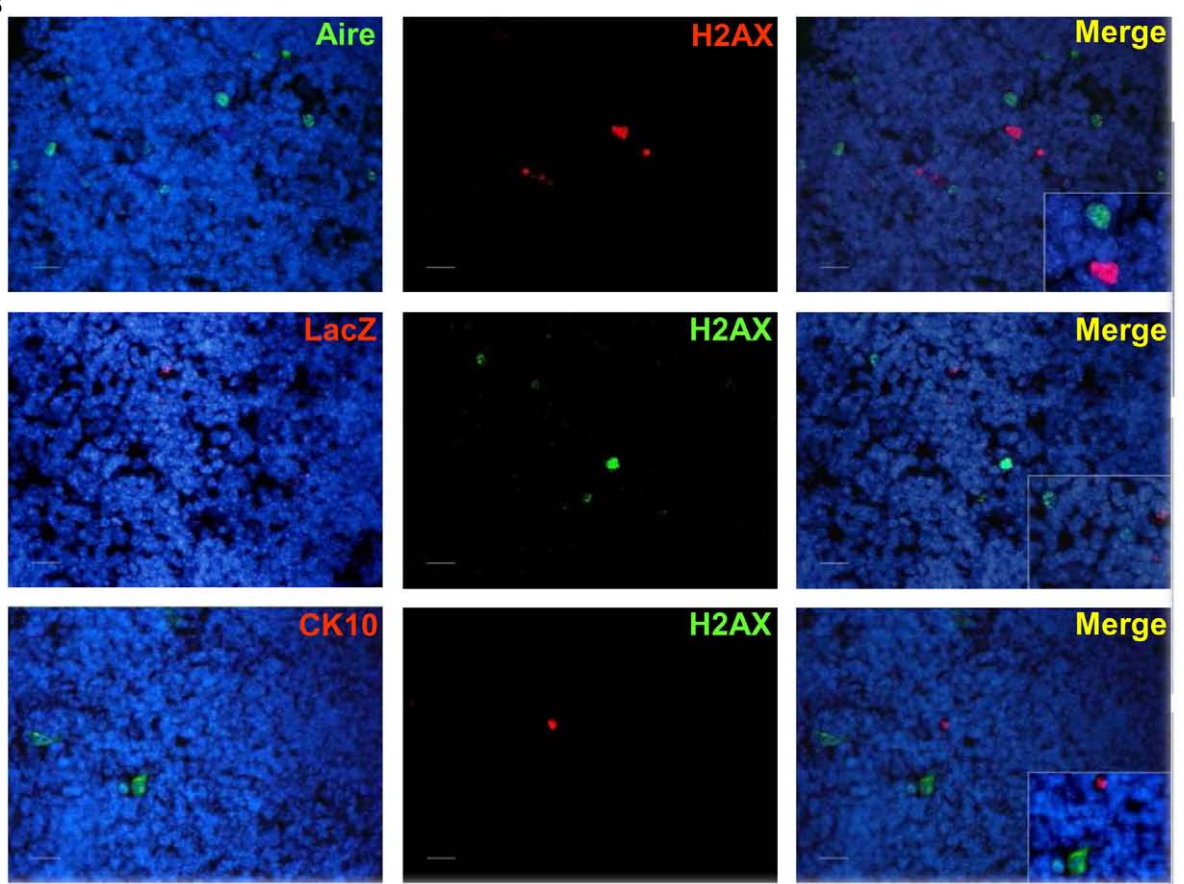

FIGURE A7 | Co-staining of Aire, LacZ, and CK10 with Ki67 or phospho-H2AX in Aire+/- mouse thymus. (A) Aire, LacZ, and CK10 showed no co-localization with Ki67. (B) Aire, LacZ, and CK10 showed no co-localization with phospho-H2AX. DAPI was used for nuclear staining Shown are representatives of at least two experiments. Bars correspond to $40 \mu \mathrm{m}$. 

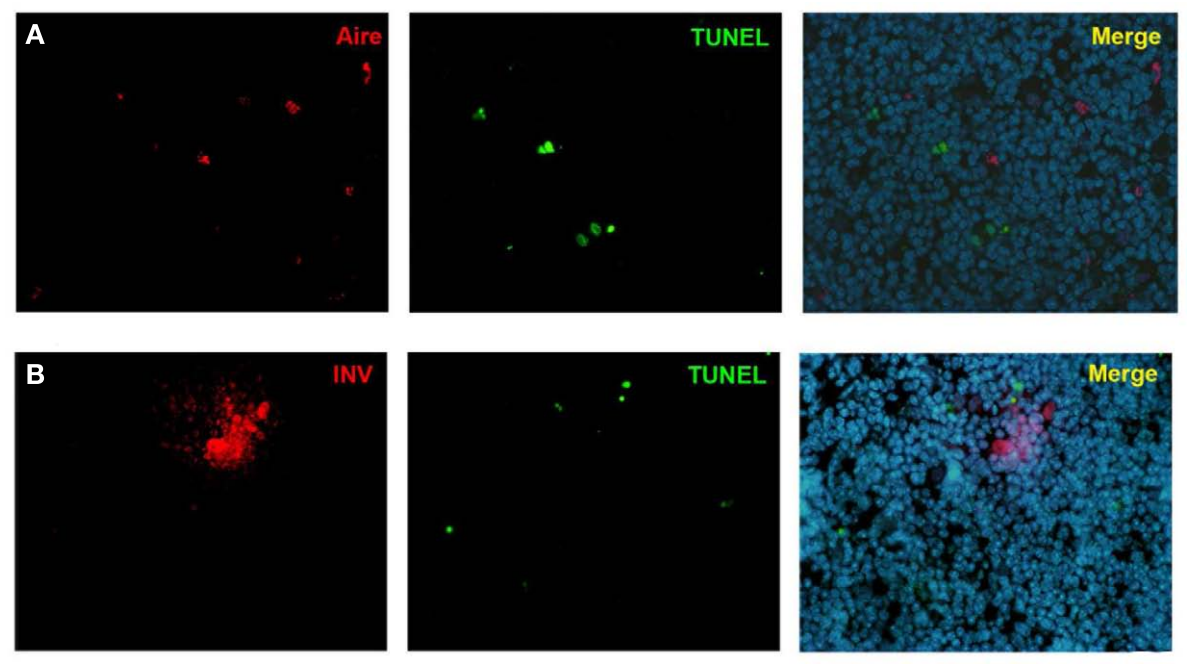

FIGURE A8 | Co-stainings of Aire, involucrin, and TUNEL in WT mice thymus. Neither Aire (A) nor involucrin (B) showed any co-localization with TUNEL positive cells. Shown are representatives of two experiments.
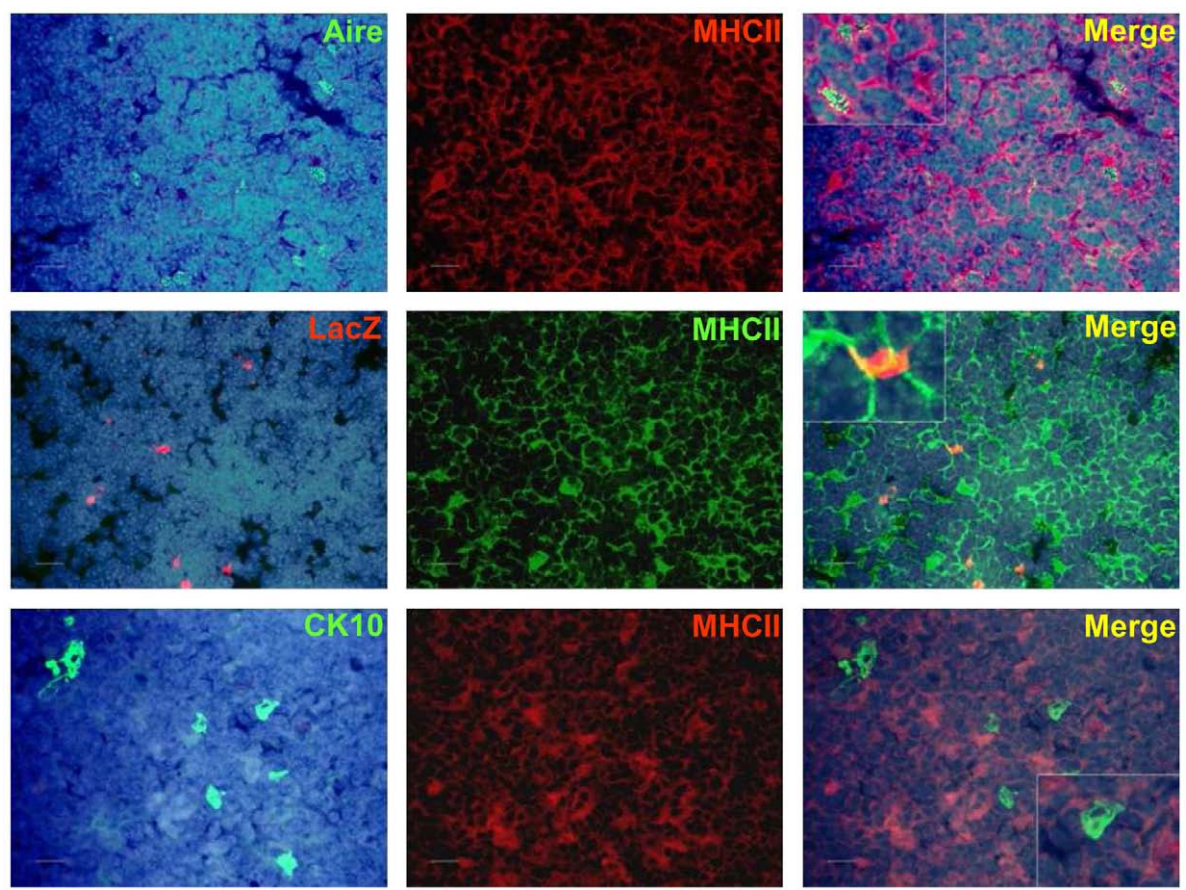

FIGURE A9 | Co-staining of Aire, LacZ, and CK10 with MHCII in Aire+/(LacZ) and WT (Aire, CK10) mouse thymus. Aire+ cells co-stained strongly with $\mathrm{MHCll}$, whereas there was a weak co-staining or no co-staining for
LacZ+ cells and CK10+ HCs with MHCII. DAPI was used for nuclear staining. Shown are representatives of at least two experiments. Bars correspond to $40 \mu \mathrm{m}$. 